\title{
ÉCOULEMENTS AU-DESSUS DE MACRO-RUGOSITÉS ARTIFICIELLES CONSTITUÉES DE PLOTS.
}

\author{
S. RICHARD (1), J. CHORDA (2) et M. LARINIER (3)
}

1) DDAF OISE, Service Equipement Rural, 29 Boulevard Amyot d'Inville, BP 50320 , 60021 Beauvais, France

2) Institut de Mécanique des Fluides de Toulouse, Avenue du Professeur Camille Soula, 31400 Toulouse, France.

3) CSP-CEMAGREF-GHAAPPE, Institut de Mécanique des Fluides de Toulouse, Avenue du Professeur Camille Soula, 31400 Toulouse, France.

\section{JÉSUMÉ}

Les écoulements au-dessus de macro-rugosités, dans lesquels les dimensions des éléments rugueux sont du même ordre de grandeur que les profondeurs des écoulements, trouvent de nombreuses applications dans l'hydraulique environnementale, en particulier dans les dispositifs de franchissement des obstacles pour les poissons migrateurs. Cet article présente une synthèse des résultats expérimentaux acquis lors d'essais en canal vitré menés à l'Institut de Mécanique des Fluides de Toulouse, en utilisant comme rugosités des rangées de plots cylindriques. Après une description des écoulements observés, on examine l'influence des différents paramètres. Des relations liant le débit à la pente, à la submersion relative, à la porosité transversale et à l'écartement longitudinal sont proposées. Les profils verticaux des vitesses sont présentés. Les résultats sont discutés dans une perspective piscicole et un exemple d'application dimensionnelle relative au franchissement des poissons est présenté.

Mots-clés : canal expérimental, macro-rugosité, passe à poissons, rivière de contournement.

\section{FLUME STUDY OF FLOW ABOVE LARGE-SCALE ARTIFICIAL ROUGHNESS.}

\section{ABSTRACT}

Flow above large-scale roughness, in which the size of bed elements is of the same order of magnitude as the depth of the flow, is of great interest in aquatic environment field, namely in fish passage technology. This article presents a synthesis of the experimental results of laboratory tests conducted in a flume at the Institute of Fluid Mechanics in Toulouse, using large roughness elements made of cylindrical blocks. Following a description of the observed flows, the influence of the main factors are examined and relationships between flow discharge and channel slope, relative submersion, transversal porosity and longitudinal distance between rows are proposed. Examples of vertical velocity profiles are presented. These results are discussed in a fish passage perspective and an example of dimensional application is given.

Key-words : flume, large-scale roughness, fish pass, natural bypass channel. 


\section{INTRODUCTION}

Les écoulements à fortes pentes au-dessus de macro-rugosités, dans lesquels les dimensions des rugosités sont du même ordre de grandeur que les profondeurs de l'écoulement, ont été relativement peu abordés. Ils trouvent cependant de nombreuses: applications, liées pour la plupart à des problèmes environnementaux, en particulier dans le domaine des dispositifs de franchissement des obstacles pour les poissons migrateurs. On citera les rivières de contournement, dont le principe consiste à relier biefs amont e aval par un chenal dans lequel l'énergie est dissipée et les vitesses réduites par la rugosité. du fond et des parois, et par une succession de singularités plus ou moins régulièremen réparties (LARINIER, 1992 ; DVWK, 1996 ; GEBLER, 1998). Dans certains cas, ces dispositifs de franchissement peuvent constituer des parcours d'eau vive adaptés à la pratique du canoë-kayak.

II n'existe pas de critères de dimensionnement pour ce type de dispositifs, comme i: en existe par exemple pour les passes à bassins successifs ou les passes à ralentisseurs. La conception de tels ouvrages demeurant encore basée sur le retour d'expérience, il reste difficile d'éviter, sur un projet donné, tout risque de générer des singularités (chutes, vitesses) incompatibles avec les capacités de nage des espèces migratrices considérées. Ces aménagements nécessitent quelquefois des adaptations post-construction qui peuvent s'avérer relativement importantes. Pour les biologistes et ingénieurs travaillant dans le domaine du génie piscicole, et plus spécifiquement dans le domaine des dispositifs de franchissement des barrages, la recherche dans le domaine des écoulements au-dessus des macro-rugosités est actuellement considérée comme l'une des priorités, afin de définir des critères de conception de tels dispositifs sur des bases plus rationnelles, en termes de débit, de pente, de caractéristiques des rugosités ou des singularités, suivant les espèces migratrices prises en compte (PARASIEWICZ et al., 1998).

Ces écoulements sont caractérisés par des pentes importantes, des rapports profondeur sur dimensions des rugosités faibles, induisant des déformations marquées de la surface libre. Les concepts habituels utilisés en hydraulique à surface libre deviennent difficiles à définir sinon inapplicables. La simulation numérique de tels écoulements dans une approche mécaniste fine paraît d'autre part irréaliste, compte tenu des moyens nécessaires à la description de la bathymétrie, de la difficulté à définir les conditions aux limites, et de l'aspect fondamentalement tridimensionnel des champs de vitesses.

La complexité intrinsèque de ce type d'écoulement implique le recours à l'expérimentation sur modèle réduit physique, seul moyen permettant d'obtenir une caractérisation précise de celui-ci pour des configurations géométriques données des macro-rugosités.

Cet article présente une brève synthèse des essais en canal vitré réalisés à l'Institut de Mécanique des Fluides de Toulouse (RICHARD, 1996), la présentation et la discussion des résultats expérimentaux étant volontairement orientées vers les applications possibles dans le domaine piscicole, en particulier la conception des dispositifs de franchissement pour les poissons migrateurs.

\section{MATÉRIEL ET MÉTHODES}

\section{Le canal d'essais et la métrologie}

D'une longueur de $12 \mathrm{~m}$ (largeur : $25 \mathrm{~cm}$, hauteur : $35 \mathrm{~cm}$ ), le canal vitré est inclinable de façon continue jusqu'à $10 \%$ de pente. Il est alimenté par pompage (débit réglable jusqu'à $50 \mathrm{l} / \mathrm{s}$ ), un débitmètre électromagnétique (précision $\pm 0,4 \%$ ) permettant la mesure du débit en continu. Une vanne de réglage du niveau d'eau, située en aval, permet d'obtenir un écoulement uniforme. 
La mesure des tirants d'eau pose un problème particulier de par la nature perturbée des écoulements. Les mesures de niveau à l'aide de prises de pression sur le fond du canal ne sont pas appropriées, du fait de la répartition des pressions internes fortement non hydrostatiques (veines plongeantes, courants de retour, ...) ; les mesures par sondes de type résistif ou capacitif présentent, par le caractère intrusif de ces sondes l'inconvénient majeur d'être perturbées par les sillages qu'elles génèrent, d'autant plus marqués à fortes vitesses. S'agissant de déterminer des tirants d'eau moyens dans une section en s'affranchissant des fluctuations de niveau, des mesures directes de niveau au moyen d'une lame en Plexiglas disposée perpendiculairement à l'écoulement sur toute la largeur du canal, avec lecture sur une échelle limnimétrique ont été retenues. Les mesures de vitesses ont été effectuées au moyen d'un micromoulinet hydrométrique Nixon (diamètre de l'hélice $10 \mathrm{~mm}$ ) en effectuant une intégration du signal sur une durée de 30 secondes.

\section{Les formes de macro-rugosités}

Les rugosités testées sont constituées de plots cylindriques verticaux en bois de $k=8 \mathrm{~cm}$ de hauteur (sections circulaires de diamètre $\mathrm{d}=2,8 \mathrm{~cm}$ ou rectangulaires $2,8 \mathrm{~cm} \times 2,1 \mathrm{~cm}$ ), les plots étant disposés en quinconce perpendiculairement à la ligne de pente du canal (Figure 1). Les lignes ainsi formées sont régulièrement espacées d'un pas $L=0,20$ m et $0,40 \mathrm{~m}$ (espacement relatif longitudinal $L / k=2,5$ et 5 ).

L'espacement transversal entre deux cylindres d'une même rangée détermine la porosité $\theta$ définie par le pourcentage de largeur du canal $(B)$ non obstruée par les plots :

$$
\theta=1-\Sigma \mathrm{d} / \mathrm{B} \text {. }
$$

L'influence d'une rugosité uniforme du fond a été testée en garnissant le fond $\mathrm{du}$ canal de plots cylindriques de plus petites dimensions de $1,4 \mathrm{~cm}$ de diamètre et $1,4 \mathrm{~cm}$ de hauteur régulièrement répartis entre les rangées de plots décrites ci-dessus. Les montages exempts de petits plots sont dits à " fond lisse ".

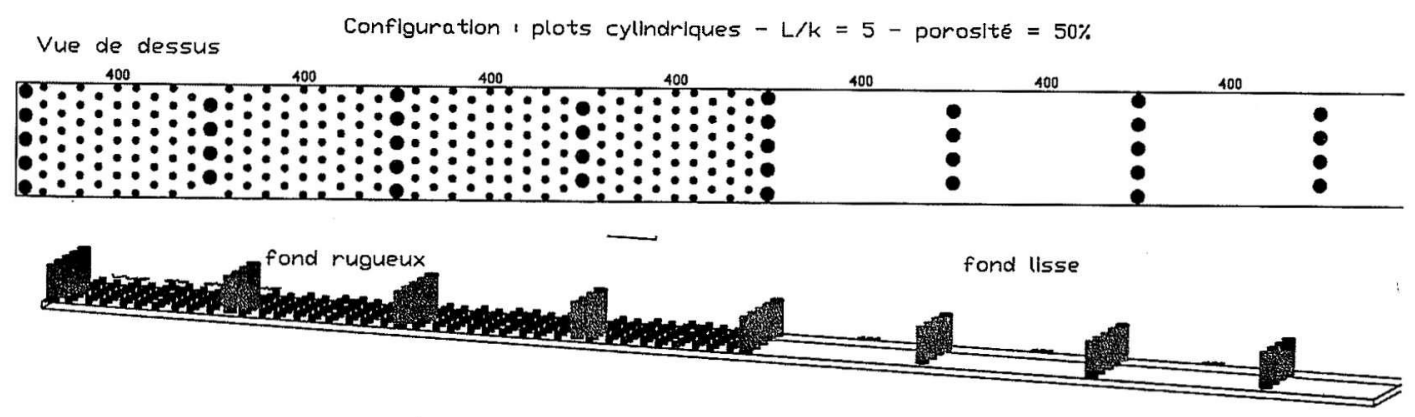

le motif de base
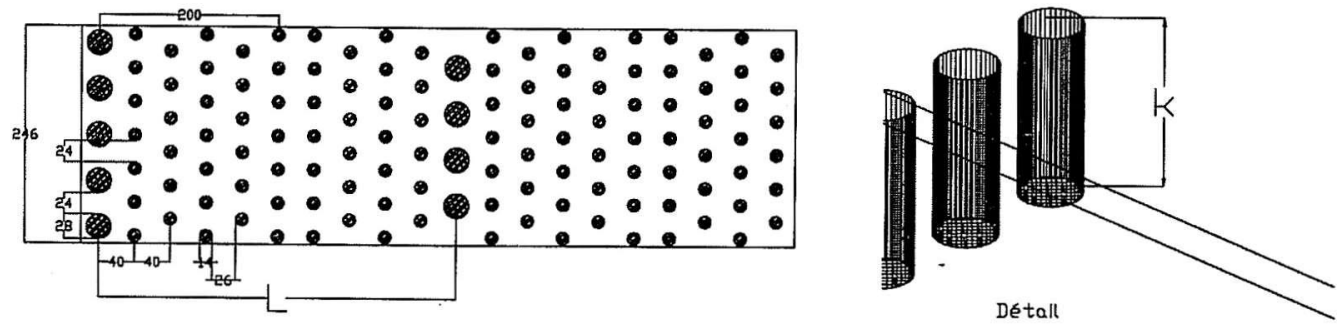

Figure 1

Disposition générale des rugosités de fond testées : plots cylindriques $L / k=5-$ porosité $=\mathbf{5 0} \%$.

\section{Figure 1}

General arrangement of the bottom roughness : cylindrical blocks L/k $=5-$ porosity $=50 \%$. 


\section{Les grandeurs mesurées}

Les mesures ont été réalisées en régime permanent dans un secteur du canc: présentant un écoulement établi « cycliquement uniforme ", c'est-à-dire dont les caractéristiques (hauteurs, vitesses) sont identiques en moyenne dans des section: distantes du pas d'espace L. Les mesures sont effectuées dans l'axe du canal. Pour unt configuration de rugosité donnée, les variables imposées sont la pente du canal (I) et k débit $(Q)$.

Les écoulements étudiés présentent des déformations importantes de la surfac libre, variables en fonction du débit et des situations d'essais. La localisation des section: de mesures conditionne la représentativité des données recueillies. Les mesures de: hauteurs d'eau moyennes ont été effectuées sur deux sections : la section située à l'amon. immédiat de la rangée de plots (hauteur h), qui permet de calculer une vitesse débitante représentative, et la section où la hauteur d'eau moyenne est minimale (hauteur $h_{m n}$ ), qu permet d'avoir une idée des distorsions de la surface libre.

Les mesures des profils verticaux des vitesses ( $u$ ), moyennées dans le temps su: 30 secondes, ont été effectuées pour trois débits correspondant à des hauteurs d'eau $\grave{c}$ l'amont des plots identiques ( $h=k, 1,5 \mathrm{k}$ et $2 \mathrm{k}$ ), pour toutes les pentes et les configurations géométriques testées. Deux sections de mesures ont été explorées : à l'amont immédiat de la rangée de plots et à mi-distance entre deux rangées de plots, correspondant dans de nombreux cas à la section de hauteur d'eau minimale.

\section{RÉSULTATS}

\section{Nomenclature des essais}

Les essais ont été réalisés pour des pentes variant de $0,5 \%$ à $7 \%$, des porosités de $42 \%$ à $75 \%$, des espacements $L / k$ de 2,5 et 5 , et des submersions relatives $h / k$ variant de 0,2 à 3 (Tableau I et Figure 2).

\section{Tableau I}

Formes et dispositions des rugosités testées.

\section{Table I}

Shapes and arrangements of the tested bottom roughness.

\begin{tabular}{cccccc}
\hline Fond & Forme & Porosité & L/K & $\begin{array}{c}\text { Disposition } \\
\text { plots/rangée }\end{array}$ & Pente I \\
\hline rugueux & circulaire & $50 \%$ & 5 & $5-4$ & $0,5-1-3-5-7 \%$ \\
lisse & circulaire & $50 \%$ & 5 & $5-4$ & $0,5-1-3-5-7 \%$ \\
rugueux & circulaire & $50 \%$ & 2,5 & $5-4$ & $1-3-5-7 \%$ \\
lisse & circulaire & $50 \%$ & 2,5 & $5-4$ & $1-3-5-7 \%$ \\
lisse & circulaire & $75 \%$ & 2,5 & $3-2-2-2$ & $1-3-5-7 \%$ \\
lisse & rectangulaire & $50 \%$ & 2,5 & $5-4$ & $1-3-5-7 \%$ \\
lisse & rectangulaire & $42 \%$ & 2,5 & $5-4$ & $1-3-5-7 \%$ \\
lisse & rectangulaire & $62 \%$ & 2,5 & $5-4$ & $1-3-5-7 \%$ \\
\hline
\end{tabular}


1. elreulalre - Porosite $50 \%-L / k=5$ - fond rugueux

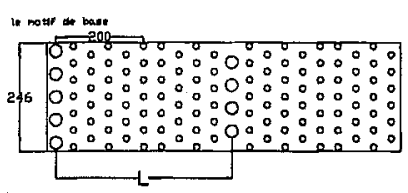

2 . Alrculalres- Porosite $50 \%-L / k=5-$ fond llsse

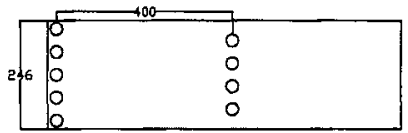

3 1 circulaires - Porosite $50 \%-L / K=2.5-$ fond rugueux

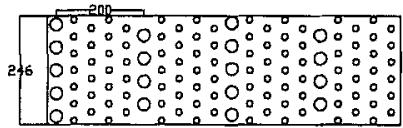

5 1 elrculalres-Porosite $75 \%-L / k=2.5$ - fond llsse

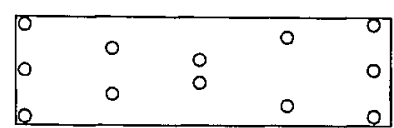

61 rectangulalres- Poroslte $50 \%-L / K=2.5-$ fond $I$ sse

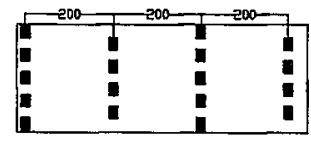

7 , rectagulalres- Poros 1 te $62 \%-\mathrm{L} / \mathrm{K}=2.5-$ fond llsse

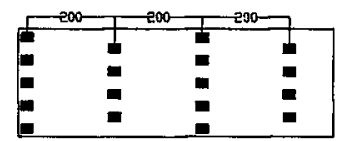

8 . rectangulaires- Porosite $42 \%-L / K=2.5$ - fond llsse

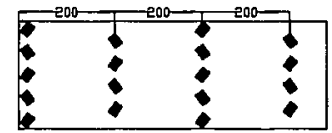

\section{Figure 2}

Vues en plan des formes et dispositions testées.

\section{Figure 2}

Plane views of the tested shapes and patterns.

\section{Description des écoulements}

Pour la pente de $0,5 \%$, l'écoulement est fluvial (ou subcritique) quels que soient le débit et l'espacement $L / k$. Pour la pente de $1 \%$, on se trouve à la limite entre le régime fluvial et transcritique ou "en cascade ", caractérisé par une succession de chutes et de ressauts. Pour les valeurs élevées du débit, le régime devient torrentiel (supercritique). La même évolution est observée pour la pente $3 \%$, le régime transcritique étant plus marqué (Figure 3). Pour les porosités transversales $62 \%$ et $75 \%$, on observe même à faible débit les caractéristiques du régime transcritique. Pour les pentes de $5 \%$ ou $7 \%$ et quelle que soit la porosité, le régime fluvial n'est jamais observé.

Le passage du régime "en cascade " au régime torrentiel intervient pour des submersions relatives $\mathrm{h} / \mathrm{k}$ comprises entre 1,2 et 1,3 . Lorsque l'écoulement est dans la zone de transition entre les régimes transcritique et rapide, le conflit entre les jets plongeants et les jets de surface entraîne la formation d'ondes déferlantes caractérisées par un fort balancement latéral qui s'amplifie vers l'aval. 

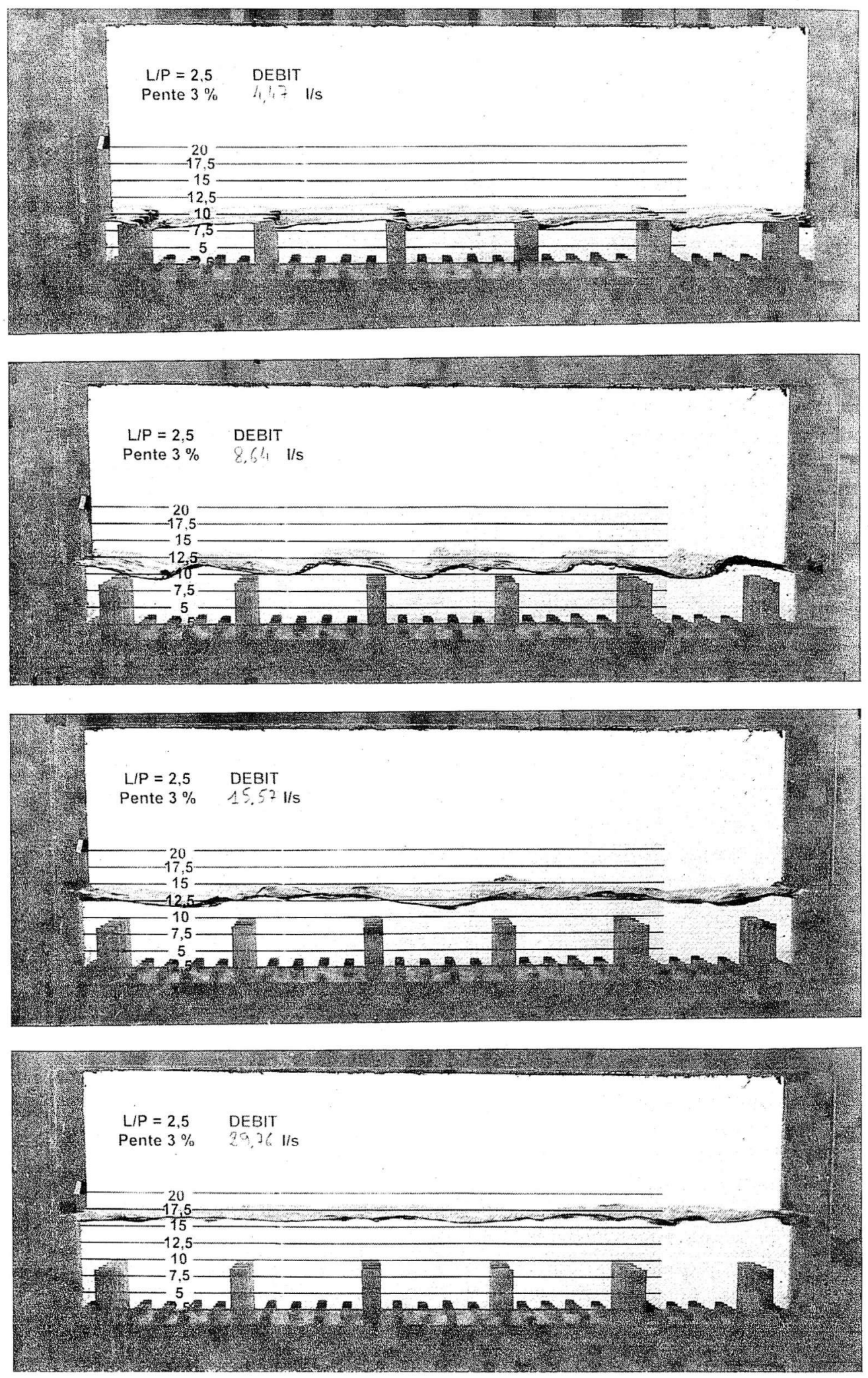

Figure 3

Vue de l'écoulement pour différents débits $-L / k=2,5$, pente $3 \%$.

\section{Figure 3}

View of flow for different discharges $-L / k=2.5$, slope $3 \%$. 


\section{Variables adimensionnelles}

Le recours à des variables sans dimensions présente l'intérêt d'une représentation unifiée des résultats avec comparaison possible des facteurs d'influence, et permet l'établissement de courbes et de formules facilement transposables à des cas particuliers.

A partir des variables suivantes:

B : largeur du canal $(\mathrm{m})$;

1 : pente du canal ;

g : accélération de la pesanteur $\left(9,81 \mathrm{~m} / \mathrm{s}^{2}\right)$;

$k$ : hauteur des rugosités $(m)$;

$Q$ : débit $\left(\mathrm{m}^{3} / \mathrm{s}\right)$;

$\mathrm{h}$ : hauteur d'eau caractéristique $(\mathrm{m})$.

L'analyse dimensionnelle permet de définir la submersion relative $h^{*}=h / k$ et un débit adimensionnel $\mathrm{q}^{\star}$ :

$$
q^{*}=Q /\left(B \cdot g^{0,5} \cdot k^{1,5}\right)
$$

La présentation des résultats sous la forme d'un débit adimensionnel (RAJARATNAM et KATOPODIS, 1990 ; CHORDA et al., 1995 ; PUEL, 1995) semble plus pertinente, et surtout d'une utilisation plus aisée, que celles, plus fréquentes, données sous la forme du rapport vitesse débitante sur vitesse de frottement $\left(U / U^{*}\right)$, d'un coefficient de Chézy adimensionnel ou d'un coefficient de frottement de Darcy-Weisbach $(\lambda)$ (MIRAJGAOKER et CHARLU, 1963 ; JUDD et PETERSON, 1969 ; BATHURST, 1978 ; THOMPSON et CAMPBELL, 1979).

Les vitesses sont également données sous la forme adimensionnelle $\left(u^{*}\right)$ obtenue en divisant la vitesse mesurée $(u)$ par la vitesse débitante $(U)$, rapport entre débit et section brute (Q/B.h) :

$$
u^{*}=u \cdot B \cdot h / Q
$$

Les profils sur une verticale sont exprimés en fonction de la profondeur relative $\mathrm{y}^{\star}=\mathrm{y} / \mathrm{h}$, où y est la distance du point de mesure par rapport au fond du canal. Cette représentation n'est pas classique en mécanique des fluides, mais présente l'intérêt de la simplicité pour les applications pratiques, en particulier pour le dimensionnement des ouvrages.

\section{Relation hauteur-débit}

La Figure 4 montre l'évolution de la submersion relative $(h / k)$ mesurée à l'amont immédiat des plots en fonction du débit $\mathrm{q}^{\star}$, pour plusieurs pentes, une porosité de $50 \%$ et $L / k=2,5$. Les courbes présentent une inflexion marquée pour une submersion relative voisine de 1,2 qui correspond à une surface libre située légèrement au-delà de la limite de crête des éléments rugueux. Les graphes des points expérimentaux se trouvent unifiés en utilisant, par analogie avec un écoulement uniforme, une débitance adimensionnelle définie par le rapport $q^{*} / \sqrt{ } \mathrm{l}$ (Figure 4).

\section{Profils de vitesses}

Les profils de vitesses pour une porosité $\theta=50 \%$ et $L / k=2,5$ en amont des rangées de plots disposés sur un fond lisse, pour trois niveaux de submersion relative $h / k=1,1,5$ et 2 , sont représentés sur la Figure 5 . Les points de mesures présentent une assez bonne cohérence sur toute l'étendue de la plage des pentes du canal testées. 
$\theta=\mathbf{5 0} \%-\mathrm{L} / \mathrm{k}=\mathbf{2 , 5}$
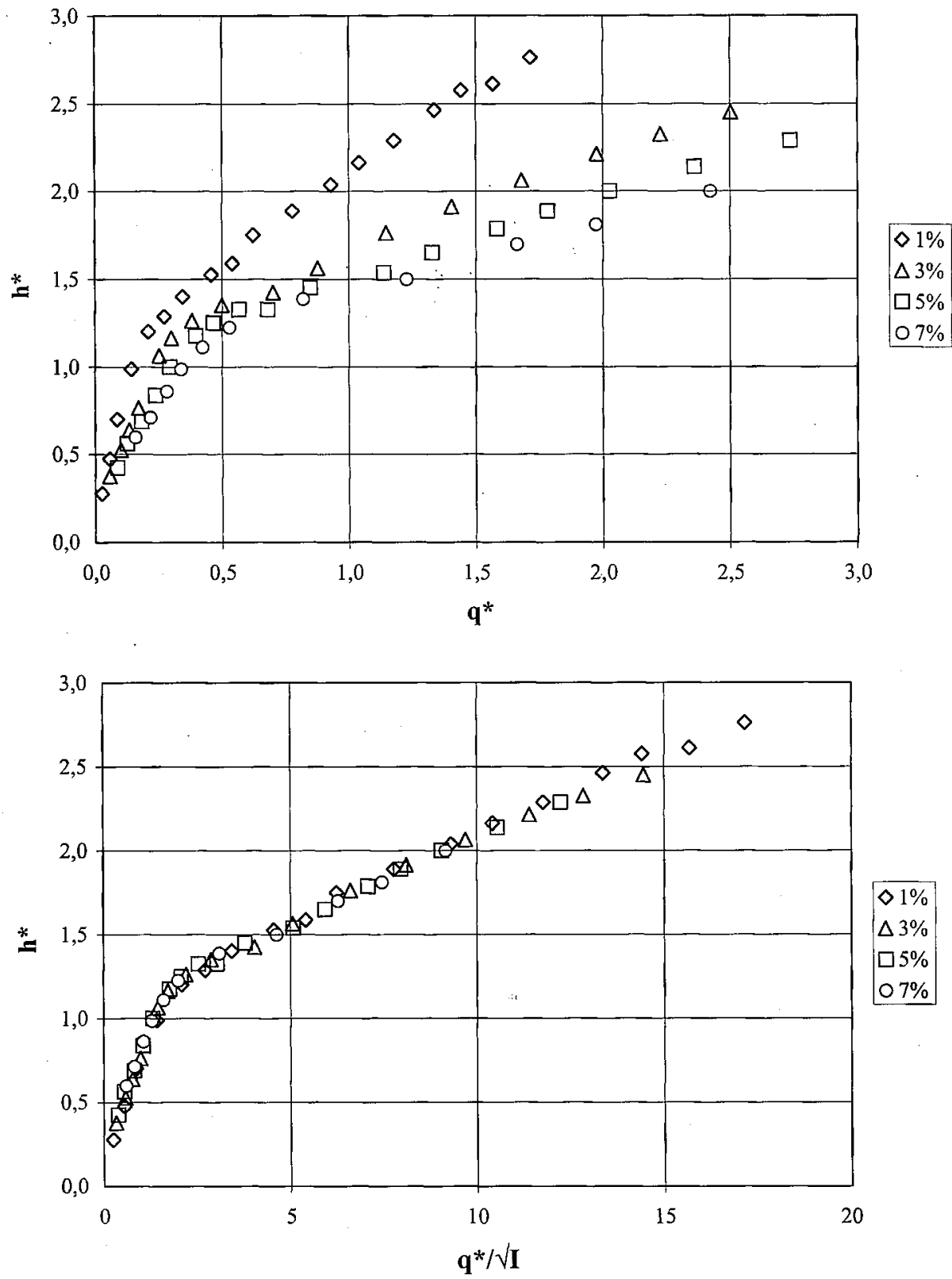

Figure 4

Submersion relative en fonction du débit adimensionnel.

\section{Figure 4}

Relative submersion versus dimensionless discharge.

Pour $h / k=1$, les profils sont relativement plats avec un maximum de l'ordre di 1,5 fois la vitesse débitante à mi-profondeur. Les profils pour $h / k=1,5$ et 2 présentent un double inflexion. Les vitesses sont relativement constantes entre le fond et $0,6 \mathrm{k}$, $\varepsilon$ comprises entre 0,5 et 0,7 fois la vitesse débitante $(U)$. Les valeurs des vitesses pour le points situés au-dessus des rangées de plots paraissent distribuées selon une ic logarithmique classique. 

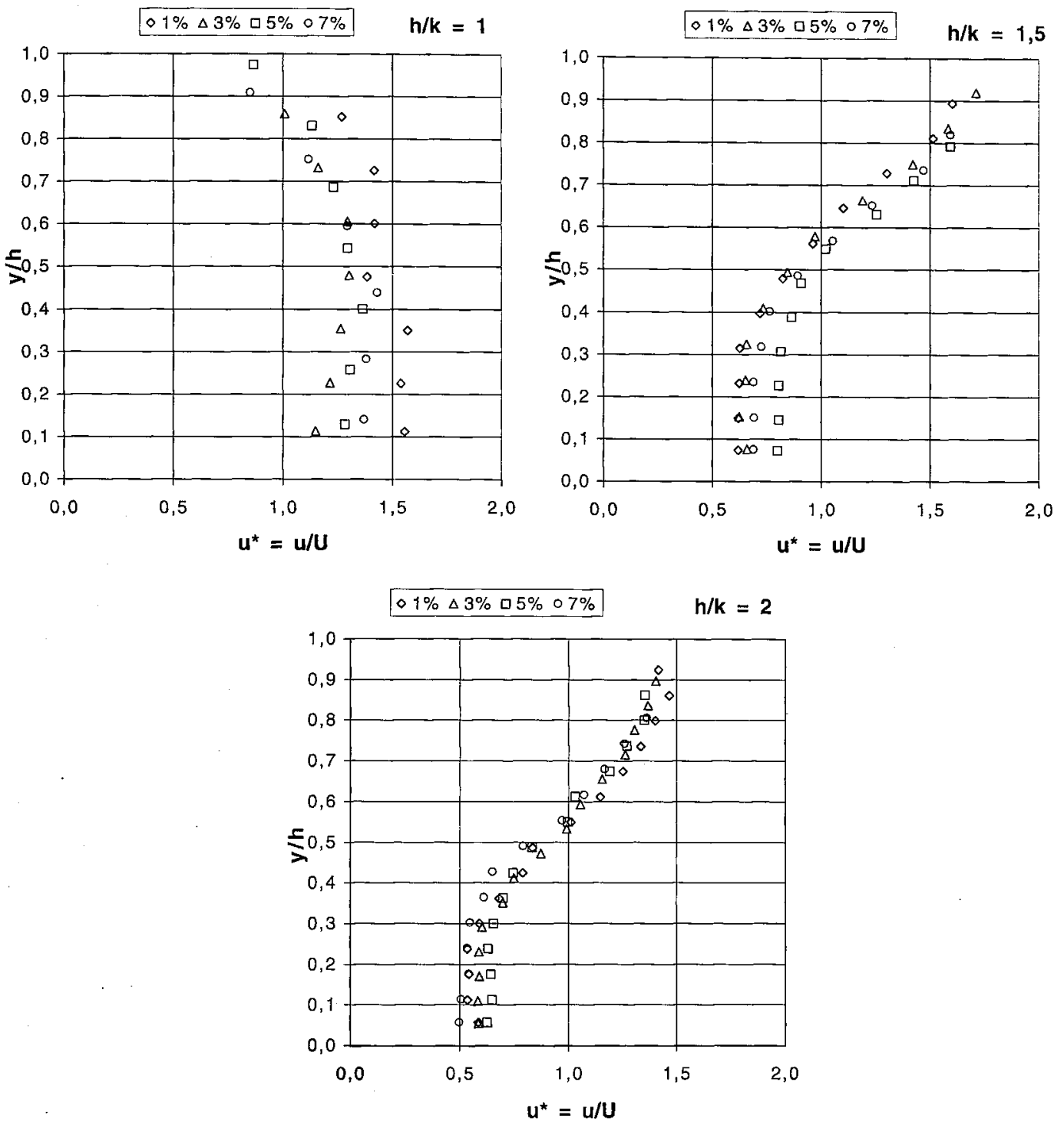

Figure 5

Profils de vitesses adimensionnels pour trois degrés de submersion - fond lisse entre les lignes de plots.

\section{Figure 5}

Dimensionless velocity profiles for three relative submersion levels - smooth bottom between the blocks lines.

\section{Influence de la configuration}

\section{Influence du fond rugueux.}

En dépit du fait que la présence des petits plots double la rugosité intrinsèque du fond, les submersions relatives observées à l'amont immédiat des plots sont sensiblement les mêmes que le fond soit lisse ou rugueux. Les écarts enregistrés sur les hauteurs d'eau minimales entre les plots sont plus importants. On note en effet des valeurs de hauteur d'eau plus importantes sur le fond rugueux, cette tendance s'accentuant avec la pente et diminuant avec la submersion relative. 
Les tirants d'eau au niveau des plots étant quasiment les mêmes avec ou sans la rugosité de fond induite par les petits plots, cela signifie que les deux échelles de rugosité ne sont pas additives : les macro-rugosités déterminent pratiquement seules la ligne d'eau en masquant les éléments sous-jacents.

L'examen des profils de vitesses ne révèle pas de différence significative pour les faibles submersions. Cependant, à $h / k=2$, la présence des petits plots semble accentuer les gradients des profils verticaux des vitesses (vitesses plus faibles au fond, et au contraire plus fortes sous la surface libre), sans pour cela modifier sensiblement la structure de l'écoulement.

\section{Influence de la forme des plots}

L'influence de la forme des rugosités (section circulaire ou rectangulaire) a été testée pour un écartement longitudinal $L / K$ de 2,5 , une porosité transversale de $50 \%$, et pour des pentes variant de $1 \%$ à $7 \%$. L'amplitude du balancement transversal observé à faible pente, ainsi qu'au changement de régime, apparaît réduite pour des plots de section rectangulaire. Les couples débit-hauteur sont équivalents. Les profils de vitesses coïncident quasiment pour les deux formes testées. La forme en plan des macro-rugosités n'est pas une variable influant sur les caractéristiques moyennes de l'écoulement.

\section{Influence de l'espacement longitudinal}

A débit égal, les valeurs de la submersion relative observée pour $L / k=5$ sont légèrement inférieures à celles observées pour $L / k=2,5$. Par ailleurs, pour $L / k=5$, les instabilités de surface ont tendance à être plus marquées et les profils de vitesses beaucoup moins homogènes lorsque la pente varie.

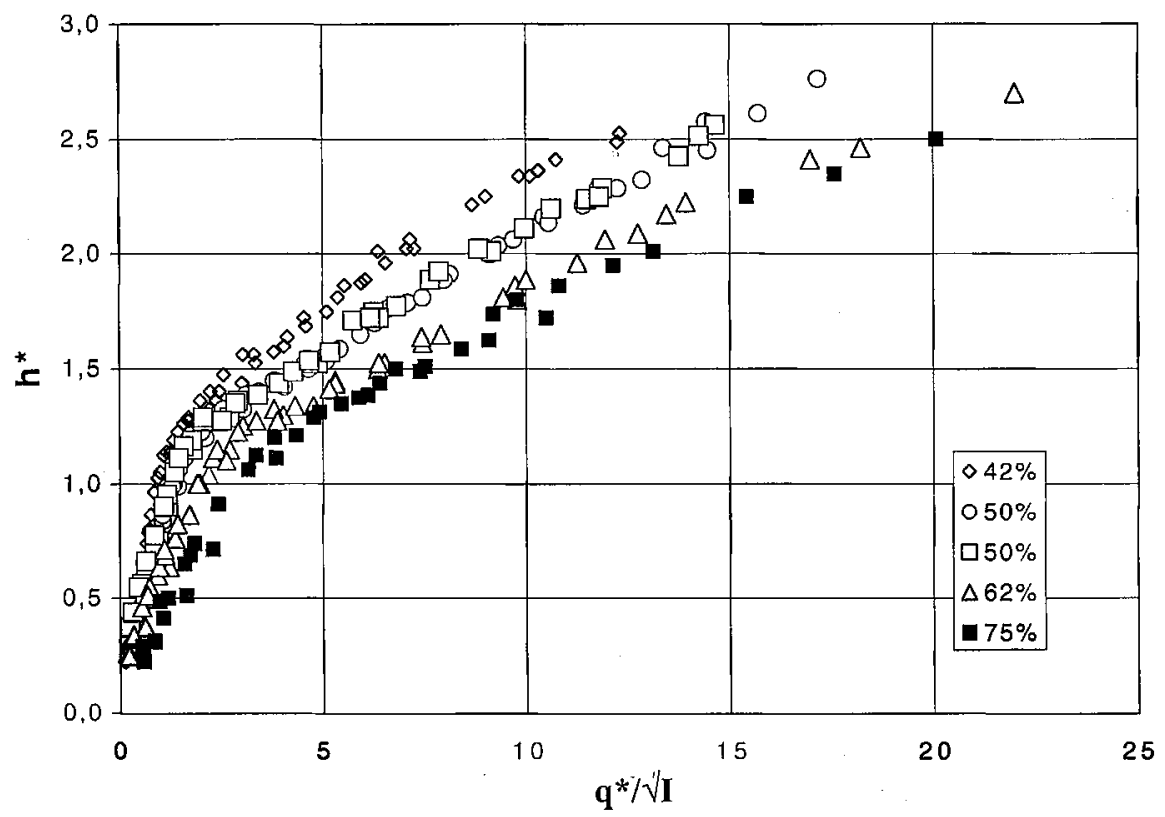

Figure 6

Influence de la porosité transversale sur la relation débit adimensionnelsubmersion relative.

\section{Figure 6}

Transverse porosity influence on the dimensionless discharge-relative submersion relationship. 
Influence de la porosité transversale et de la dispositions des plots

Les porosités transversales de $42 \%, 50 \%, 62 \%$ et $75 \%$ ont été testées avec un écartement longitudinal relatif $\mathrm{L} / \mathrm{k}$ égal à 2,5. La Figure 6 présente la submersion relative en fonction de la débitance adimensionnelle. A débitance égale, la submersion relative décroît quand la porosité transversale croît : une augmentation de l'ordre de $20 \%$ de la porosité se traduit par un abaissement de la ligne d'eau voisin de $20 \%$.

L'effet de la porosité sur les profils de vitesses est bien marqué : en abaissant celle-ci de $50 \%$ à $42 \%$, soit de $12 \%$, les vitesses sont réduites de $50 \%$ dans la partie basse du profil. Les vitesses observées dans une section médiane entre deux rangées de plots sont toutefois similaires pour les deux porosités considérées.

La plupart des essais ont été réalisés avec des montages où les plots sont disposés en quinconce par rangées successives. Tout en conservant le même nombre de plots (ou la même densité), la disposition de ceux-ci a été modifiée, en doublant le nombre de rangées et en réduisant le nombre de plots par rangée. A débit égal, cette modification de l'arrangement a pour effet de réduire significativement la submersion relative (de l'ordre de 15 à $20 \%$ d'écart). La disposition des plots, qui détermine la porosité, est un paramètre important de la résistance à l'écoulement.

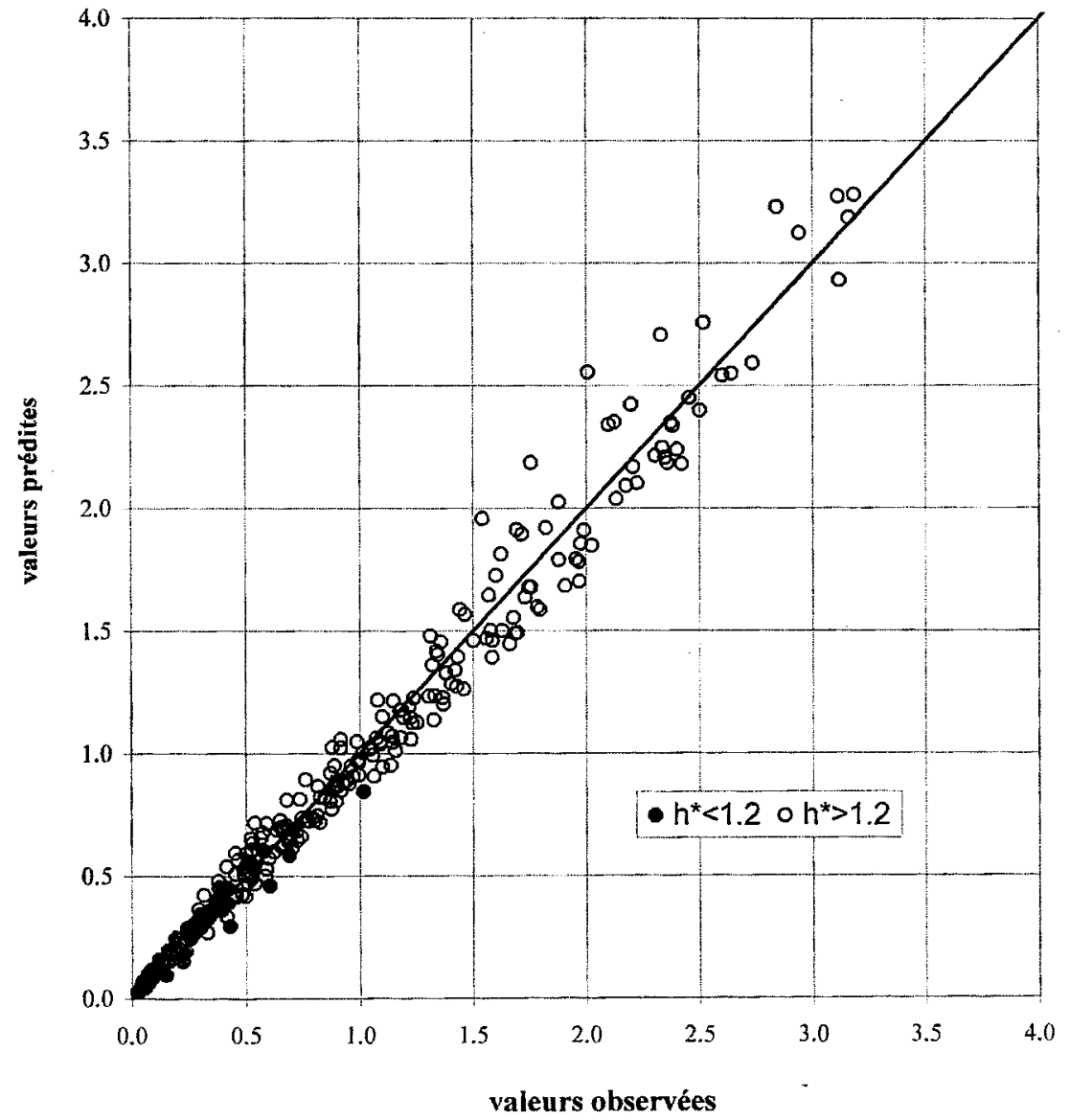

Figure 7

Relation submersion relative-débit adimensionnel : comparaison des valeurs observées et prédites.

\section{Figure 7}

Relative submersion-dimensionless discharge relationship : comparison of predicted and observed values. 


\section{Lois d'écoulements}

Les résultats ont été exprimés sous forme de relations puissances donnant le débit adimensionnel en fonction de la submersion, de la porosité, du rapport $L / k$ et de la pente. La discontinuité observée sur les nuages de points $\left(q^{*}, h^{\star}\right)$ pour $h / k=1,2$ nécessite de considérer séparément les deux régions d'écoulement:

$$
\begin{array}{lll}
h / k<1,2 & q^{*}=2,64(h / k)^{1,36}(L / k)^{0,42} \theta^{1,90} \mid 0,42 & \left(R^{2}=0,94\right) ; \\
h / k>1,2 & q^{*}=2,\left.95(h / k)^{2,52}(L / k)^{0,21} \theta^{1,34}\right|^{0,49} & \left(R^{2}=0,94\right) .
\end{array}
$$

On a porté sur la Figure 7 les valeurs prédites en fonction des valeurs mesurées.

Des relations équivalentes ont été établies, donnant le débit en fonction de la submersion minimale $\left(h_{\text {min }} / k\right)$ (RICHARD, 1996).

\section{DISCUSSION}

L'étude en canal de l'écoulement au-dessus de macro-rugosités constituées par des rangées de plots régulièrement espacés a mis en évidence trois formes d'écoulement suivant la pente et le débit. Pour la pente de $0,5 \%$, l'écoulement est fluvial, quels que soient le débit et l'espacement. Pour les pentes supérieures, on observe systématiquement; pour des faibles degrés de submersion, un écoulement « en cascade ", caractérisé par une succession cyclique de petites chutes et de ressauts : en aval de chaque élément, le fluide est accéléré (l'écoulement passe en supercritique) puis décéléré dans un ressaut hydraulique avant l'élément suivant. Les ressauts sont d'autant plus marqués que la pente est élevée et l'espacement important. On retrouve les types d'écoulements décrits par PETERSON et MOHANTY (1960), MORRIS (1968) et CHORDA et al. (1995) lors d'expérimentations portant sur des écoulements à forte pente au-dessus de barrettes ou cubes disposés sur le fond d'un canal.

Pour les valeurs élevées du débit, correspondant à un degré de submersion $h / k$ généralement supérieur à 1,3, le régime devient torrentiel. Lorsque l'écoulement est dans la zone de transition entre les régimes transcritique et rapide, on observe la formation d'ondes déferlantes qui s'amplifient vers l'aval.

L'analyse des résultats montre que les paramètres déterminants de l'écoulement sont la porosité ainsi que l'espacement longitudinal des éléments.

L'espacement influe généralement peu sur le tirant d'eau à l'amont des plots. Des expérimentations menées par ailleurs (PUEL, 1995) pour une plage de variation des espacements beaucoup plus large $(2,5<L / k<20)$ confirment la faible influence de $L / k$. Par contre, les instabilités de surface ont tendance à être plus marquées lorsque les plots sont plus éloignés. L'espacement intervient aussi très nettement sur la forme de l'écoulement entre les plots et sur la valeur du débit de transition de l'écoulement transcritique à torrentiel : lorsque le débit augmente, l'écoulement a tendance à passer plus rapidement en torrentiel pour les faibles espacements, ceci pouvant s'expliquer par le fait que l'écoulement est plus enclin à glisser au-dessus des éléments lorsqu'ils sont rapprochés, l'espace entre deux rangées de plots n'étant pas suffisant pour permettre la formation d'un ressaut.

La forme des éléments ne semble pas influer sur l'écoulement, ce qui rejoint les observations effectuées par HERBICH et SHULITS (1964). La disposition des éléments joue par contre un rôle important, qui peut s'interpréter en termes de porosité transversale (ou de taux d'obstruction). 


\section{APPLICATION PRATIQUE}

Les conditions d'écoulement en régime fluvial conviennent évidemment au poisson, mais elles ne sont rencontrées qu'à des pentes trop faibles (inférieures à $1 \%$ ) pour présenter un quelconque intérêt pratique pour la conception d'un dispositif de franchissement.

Le régime transcritique est le domaine privilégié des dispositifs de franchissement, à la condition que les ressauts ne soient pas trop violents, et que les vitesses et les tirants d'eau à l'amont immédiat de ces ressauts restent compatibles avec les capacités de nage des espèces concernées. Les chutes locales et l'intensité du ressaut ayant tendance à diminuer parallèlement à la pente, il sera possible d'augmenter l'espacement relatif des rangées lorsque la pente diminue. Pour que le régime transcritique ne soit trop marqué, le produit de la pente par l'espacement relatif doit rester limité, inférieur à une valeur que l'on peut estimer à 0,30 , par analogie avec les écoulements au-dessus de barrettes (LARINIER et CHORDA, 1995).

Le domaine des écoulements torrentiels n'est généralement pas adapté au franchissement du poisson, les vitesses devenant trop élevées dans la couche superficielle de l'écoulement au-dessus des plots. Le poisson a néanmoins la possibilité de progresser au niveau du fond où les vitesses restent relativement homogènes et beaucoup plus limitées.

Les résultats des essais peuvent être utilisés pour la conception des dispositifs de franchissement pour les poissons migrateurs. Ils permettent, pour une configuration en similitude géométrique avec les configurations testées (espacement longitudinal, porosité, pente), d'apprécier les conditions hydrauliques dans le dispositif : type d'écoulement (fluvial, transcritique ou torrentiel), tirants d'eau, débit par mètre de largeur, allure des profils et ordre de grandeur des vitesses. Les vitesses, ainsi que les puissances dissipées volumiques doivent être adaptées aux capacités de nage des espèces considérées (LARINIER, 1992). La valeur de la puissance dissipée volumique est en effet indicatrice du niveau d'agitation et d'aération dans un écoulement et est couramment utilisée lors du dimensionnement des passes à poissons. Elle peut s'écrire, dans le cas présent :

$$
P / N=\text { p.g.q.I/h } \quad\left(\text { en Watts } / m^{3}\right)
$$

On a donné, à titre d'exemple, les caractéristiques hydrauliques d'un écoulement au-dessus de plots de hauteur $0,50 \mathrm{~m}$ et de diamètre $0,18 \mathrm{~m}$, disposés en rangées espacées de $1,25 \mathrm{~m}(L / k=2,5)$, plots en similitude géométrique par rapport à ceux testés $(1 / 12,5)$.

La relation entre le débit unitaire $(q)$ et le tirant d'eau (h) à l'amont immédiat des plots est donnée à la Figure 8 . On a porté sur le même graphique la puissance dissipée volumique.

Les profils de vitesses caractéristiques en amont des plots pour $h / k=1$ et $h / k=1,5$ sont donnés sur la Figure 9. Pour $h / k=1,5$, les vitesses entre les plots atteignent $2,3 \mathrm{~m} / \mathrm{s}$ en surface alors qu'elles restent inférieures à $1 \mathrm{~m} / \mathrm{s}$ entre les plots. Entre ces deux valeurs de la submersion relative, les débits unitaires varient de $0,250 \mathrm{~m}^{3} / \mathrm{s} / \mathrm{m}$ à plus de $0,750 \mathrm{~m} 3 / \mathrm{s} / \mathrm{m}$, et la puissance dissipée volumique entre 125 watts $/ \mathrm{m}^{3}$ et 300 watts $/ \mathrm{m}^{3}$. 


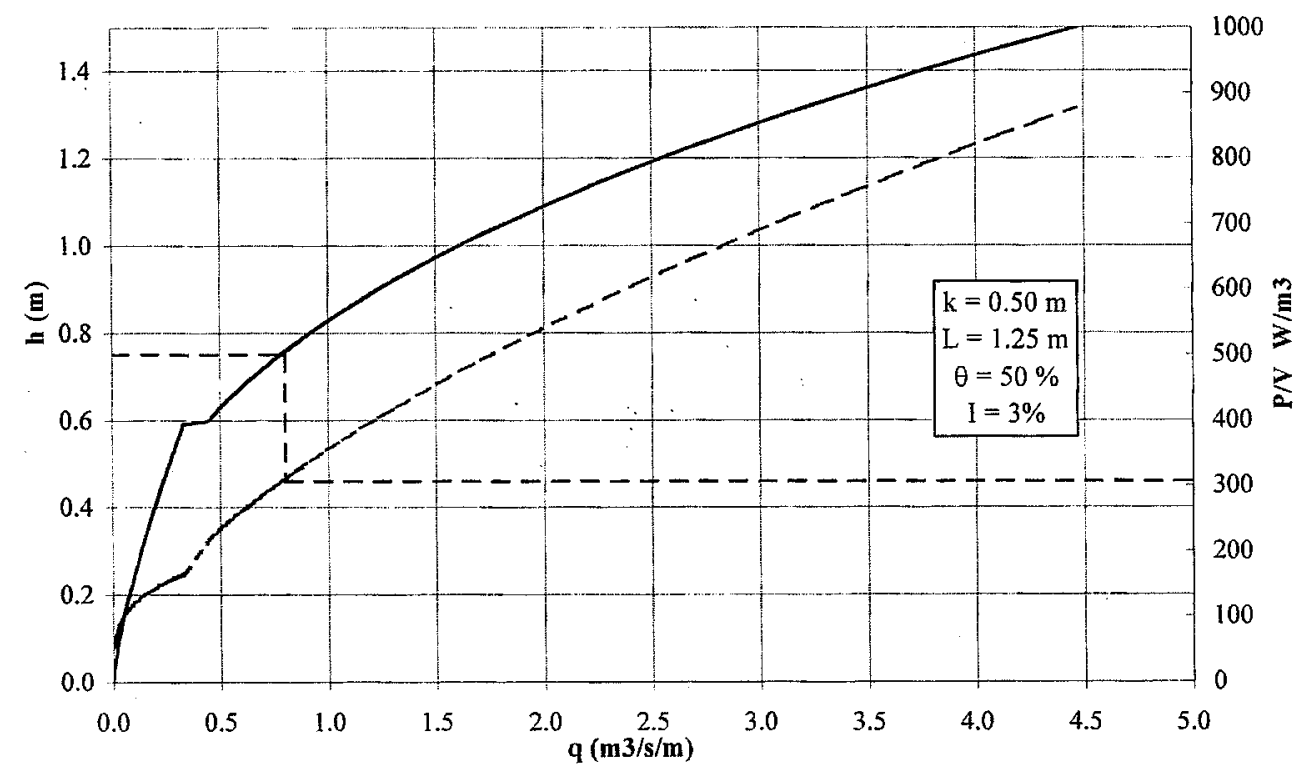

Figure 8

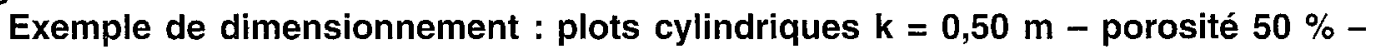
$L / k=2,5-I=3 \%$ : débits, puissances dissipées volumiques.

Figure 8

Field application example : cylindrical blocks $k=0.50 \mathrm{~m}$ - porosity $50 \%-$ $L / K=2.5-I=3 \%$ : discharge and volumetric dissipated power.

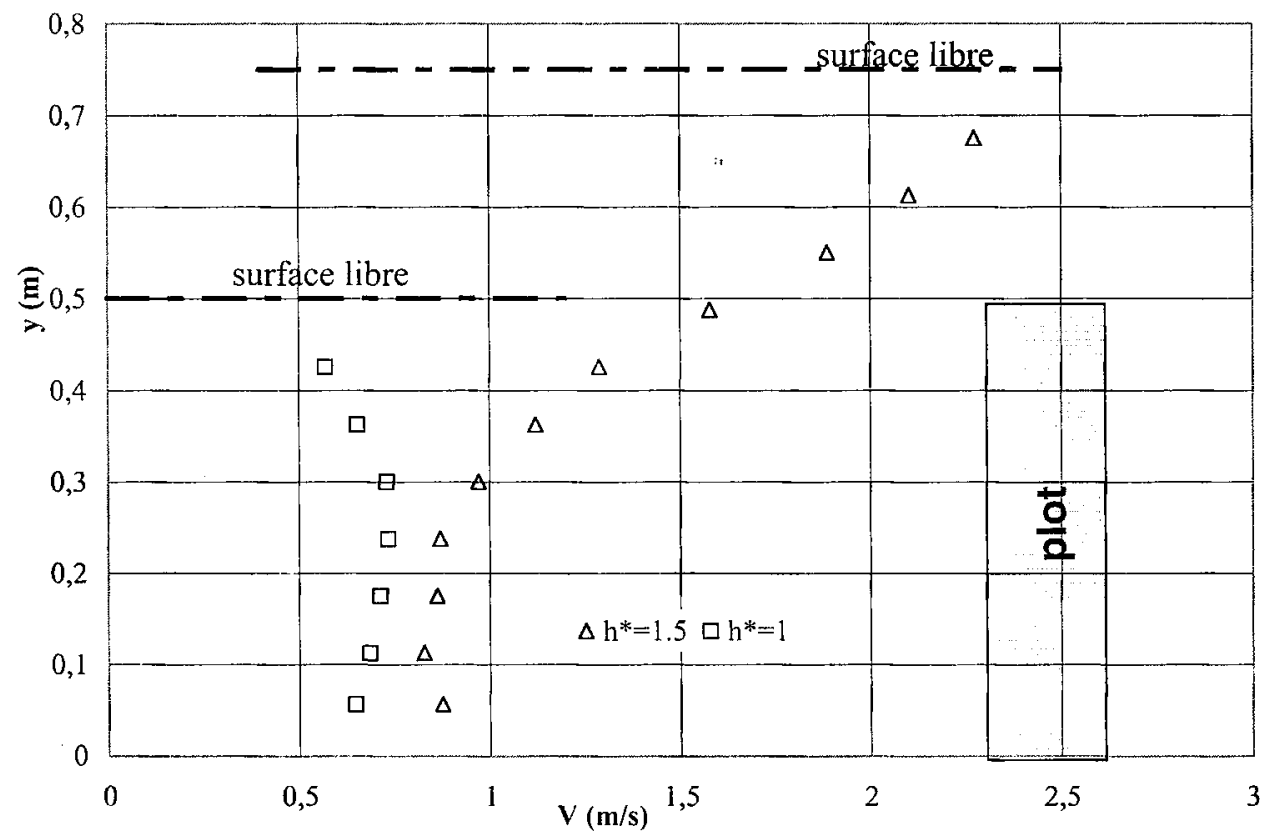

Figure 9

Exemple de dimensionnement : plots cylindriques $\mathrm{k}=0,50 \mathrm{~m}$ - porosité $50 \%$ $L / k=2,5-I=3 \%$ : profils de vitesses.

Figure 9

Field application example : cylindrical blocks $k=0.50 \mathrm{~m}$ - porosity $50 \%-$ $L / k=2.5-I=3 \%$ : velocity profiles. 


\section{CONCLUSION}

La présente étude montre que l'utilisation de rugosités disposées en rangées permet théoriquement l'aménagement de dispositifs de franchissement dans lesquels les conditions d'écoulement sont compatibles avec les capacités de nage des espèces migratrices.

Ces essais ne constituent cependant qu'une première étape d'une recherche plus générale dans laquelle devront être étudiées d'autres configurations géométriques a priori plus adaptées à l'aspect piscicole du problème et prenant en compte les contraintes constructives : porosités des éléments rugueux variables en fonction de la profondeur, présence de seuils de fond pour limiter la puissance volumique dissipée, formes des macro-rugosités plus naturelles, variabilité des espacements plus importante. On fera porter les efforts sur l'influence du facteur pente dans ce type d'écoulement, sachant que c'est la faiblesse de la pente des rivières de contournement qui, en rendant souvent difficile leur intégration au niveau des barrages, en limite actuellement la généralisation.

\section{BIBLIOGRAPHIE}

BATHURST J.C., 1978. Flow resistance of large-scale roughness. Proceedings ASCE, Journal of the Hydraulics Division, 104, 1587-1603.

CHORDA J., LARINIER M., FONT S., 1995. Le franchissement par les poissons migrateurs des buses et autres ouvrages de rétablissement des écoulements naturels lors des aménagements routiers et autoroutiers. Etude expérimentale, Rapport GHAAPPE 95/03-HYDRE 159, $17 \mathrm{p}$.

DVWK, 1996. Fishaufstieganlagen - Bemessung, Gestaltung, Funktionskontrole. DVWK-Merkblatt 232, Bonn, ISBN 3-89554-027-7, 100 p.

GEBLER R., 1998. Examples of near-natural fish passes in Germany : drop structures conversions, fish ramps, and bypass channels. In : JUNGWIRTH M., SCHMUTZ S. and WEISS S. (Eds.), Fish migration and fish bypasses, Fishing New Books, 403-419.

HERBICH J.B., SHULITS S., 1964. Large-scale roughness in open-channel flow. Proceedings ASCE, Journal of the Hydraulics Division, 90, 203-230.

JUDD H.E., PETERSON D.F., 1969. Roughness of large bed element streams. Annual Meeting ASCE, Logan, Utah, $14 \mathrm{p}$.

LARINIER M., 1992. Passes à bassins successifs, prébarrages et rivières artificielles. Bull. Fr. Pêche Piscic., 326-327, 45-72.

LARINIER M., CHORDA J., 1995. Prise en compte de la migration du poisson lors de la conception des ouvrages de rétablissement des écoulements naturels dans les aménagements routiers et autoroutiers. Rapport GHAAPPE 95/01, $16 \mathrm{p}$.

MIRAJGAOKER A.G., CHARLU K.L.N., 1963. Natural roughness effects in rigid open channels. Proceedings ASCE, Journal of the Hydraulics Division, 89, 29-44.

MORRIS H.M., 1968. Hydraulics of energy dissipation in steep, rough channels. Virginia Polytechnic Institute Research Division, Blacksburg, 108 p.

PARASIEWICZ P, EBERSTALLER J., WEISS S., SCHMUTZ S., 1998. Conceptual guidelines for nature-like bypass channels. In : JUNGWIRTH M., SCHMUTZ S. and WEISS S. (Eds.), Fish migration and fish bypasses, Fishing New Books, 348-362.

PETERSON D.F., MOHANTY P.K., 1960. Flume studies of flow in steep, rough channels. Proceedings ASCE, Journal of the Hydraulics Division, 86, 55-76. 
PUEL P., 1995. Ecoulements à surface libre au-dessus de macro-rugosités. GHAAPPE IMFT, INPT, Rapport de D.E.A. de Physique et Chimie de l'Environnement, $50 \mathrm{p}$.

RAJARATNAM N., KATOPODIS C., 1990. Hydraulics of culvert fishways III : weir baffle culvert fishways. Canadian Journal of Civil Engineering, 17, 558-568.

RICHARD S., 1996. Etude des caractéristiques des écoulements à fortes pente: au-dessus de macro-rugosités. Rapport GHAAPPE 96.04, $57 \mathrm{p}$.

THOMPSON S.M., CAMPBELL P.L., 1979. Hydraulics of a large channel paved witl boulders. Journal of Hydraulic Research, 17, 341-354. 